\title{
Predicción del impacto del cambio temporal en el uso del suelo sobre cuencas hidrológicas de alta pendiente en Costa Rica
}

\author{
Forecasting the impact of temporal land use change \\ on steep terrain watersheds in Costa Rica
}

Maikel Méndez-Morales'

Fecha de recepción: 3 de setiembre del 2012 Fecha de aprobación: 29 de noviembre del 2012

Méndez-Morales, M. Predicción del impacto

del cambio temporal en el uso del suelo sobre cuencas hidrológicas de alta pendiente en Costa Rica. Tecnología en Marcha. Vol. 26, № 3. Pág I3-25 


\section{Palabras clave}

Hidrología; IDRISI; Markov; modelación; SWMM

\section{Key words}

Hydrology; IDRISI; Markov; modeling; SWMM

\section{Resumen}

Para predecir el impacto del cambio temporal en el uso del suelo hasta el año 2025 sobre las cuencas hidrológicas del río Toyogres y la quebrada Zopilote en Costa Rica, se utilizó el modelo de simulación simultánea de cambio de uso/cobertura del suelo (MSSCUS) basado en las cadenas de Markov, Autómata Celular (AC) y Evaluación multicriterio/ multiobjetivo (EMC). El modelo hidrológico SWMM se calibró y validó con tormentas reales captadas durante 20 I para que sirvieran de plataforma del análisis hidrológico. Los registros históricos utilizados tanto en MSSCUS como en SWMM se fundamentaron en fotografías áreas de la misión TERRA de 1997 e imágenes satelitales multiespectrales del satélite WorldView-2 tomadas en $201 \mathrm{l}$.

Con base en las proyecciones arrojadas por ambos modelos, se pudo determinar que para 2025, el aumento sobre el caudal pico para una tormenta de alta intensidad (volumen máximo de 53,56 mm e intensidad máxima de $120 \mathrm{~mm} / \mathrm{hr} / 5 \mathrm{~min}$ ) sería de alrededor del 15\% para el cauce del río Toyogres y del $26 \%$ para el cauce de la quebrada Zopilote, siendo este último de gran relevancia en virtud de su limitada capacidad hidráulica. Lo anterior se da como consecuencia de un aumento del área impermeable con relación al aumento del porcentaje de área impermeable (pimp), parámetro clave en los procesos de generación de escorrentía. Los usos/ objetivos que sufren mayores cambios son HDRES, MDRES, LDRES y CROPS. Independientemente de su utilidad, se requiere mayor trabajo de investigación para calibrar y validar las proyecciones arrojadas por este tipo de modelos.

\begin{abstract}
The land use/land cover change simultaneous model based on the Markov Chain Analysis, Cellular Automata (CA_MARKOV) and Multi-CriteriaEvaluation (MCE), was used to predict the impact of the land use temporal change to 2025 over the Toyogres and Zopilote rivers watersheds in Costa Rica. The hydrologic model SWMM was previously calibrated and validated using various real rainfall storms in order to serve a hydrological analysis platform. The historical records for both models were based on aerial photography form the 1997 TERRA mission and multi-spectral images taken by the WorldView-2 satellite in 2011. Based on the model's predictions/projections for 2025, an increase of around 15\% over the peak flow for the Toyogres River and 26\% for the Zopilote River would be the consequence of a high intensity rainfall storm (maximum precipitated volume of $53.56 \mathrm{~mm}$ and maximum intensity of $120 \mathrm{~mm} / \mathrm{hr} / 5 \mathrm{~min}$ ). This is particularly relevant for the Zopilote catchment due to its limited hydraulic capacity. This is a direct consequence of an increase in the impervious area, related to the SWMM's percentage-imperviousarea (pimp), which is a key parameter in the rainfallrunoff generation processes. The land uses that experimented major changes were HDRES, MDRES, LDRES and CROPS. Regardless of its final objective, further investigation is needed in order to calibrate and validate the outcomes projected by this kind of models.
\end{abstract}




\section{Introducción}

La historia de la expansión urbana indica que las áreas urbanas están dentro de los sistemas más cambiantes y dinámicos sobre la superficie de la tierra. Más allá de su importancia económica, este crecimiento conlleva un considerable impacto sobre los ecosistemas adyacentes (Yuan et al. 2005). La expansión urbana también acarrea la transformación de los usos tradicionales del suelo (por ej., agricultura, conservación, bosque) a otros de carácter meramente residencial, lo cual implica un aumento significativo del área impermeable y consecuencias hidrológicas más agudas (Brockerhoff 2000). Monitorear estos cambios a través del tiempo resulta de gran relevancia en materia de planificación urbana, especialmente con miras al impacto hidrológico futuro. El cambio temporal del uso de suelo conlleva importantes transformaciones hidrológicas, incluyendo mayores caudales pico y menores tiempos de concentración, particularmente cuando se presentan tormentas de alta intensidad.

Esto se traduce en un mayor riesgo de inundación, desbordamiento de cauces, erosión y difusión de contaminantes (Goudie, 1990, Weng, 200I). Dada la naturaleza espacialmente distribuida de los modelos hidrológicos, se requieren planteamientos capaces de tomar en cuenta estas variables y, a la vez, aprovechar los avances en materia de sistemas de información geográfica (SIG) y plataformas de sensores remotos.

En este contexto, es indispensable avanzar en el estudio y pronóstico que la evolución temporal de ciertos parámetros pueda tener sobre los coeficientes de escorrentía superficial en áreas urbanas, considerando a la vez los cambios temporales sobre el uso del suelo. Actualmente, se encuentran disponibles diversos modelos temporales de cambio de uso, denominados Modelos de Simulación Simultánea de Cambio de Uso / Cobertura de Suelo o MSSCUS (Henríquez et al., 2006), los cuales representan una poderosa herramienta de análisis espacial orientada a aspectos tales como:

- Exploración de los mecanismos que fuerzan los cambios de uso del suelo y las variables sociales, económicas y espaciales que conducen a ello.

- Proyección temporal de los potenciales impactos ambientales y socioeconómicos derivados de los cambios en el uso del suelo.
- Evaluación de la influencia de la aplicación de políticas y esquemas de manejo sobre el ordenamiento del territorio, el desarrollo y el uso del suelo.

Los modelos MSSCUS usan parámetros tales como la extensión actual de las áreas urbanas, las principales vías de comunicación, la distancia a los centros de población, bienes e insumos, las condiciones topográficas y la existencia de terrenos en situación especial (por ej., áreas de protección).

El objetivo principal de los modelos MSSCUS es la identificación de los factores físicos y socioeconómicos que determinan o condicionan la presión sobre el cambio de uso del suelo en un territorio determinado.

Uno de los modelos de cambio de uso del suelo con más investigación y desarrollo en los últimos años es el que se basa en las técnicas estocásticas de Cadenas de Markov y Autómatas Celulares (AC) (Zhou \& Liebhold, 1995). Este modelo toma en consideración algoritmos de regresión y transición espacial. Los algoritmos de regresión establecen las relaciones entre los diversos objetivos o usos del suelo y las probabilidades de cambio de ese uso, lo cual es tradicionalmente modelado como una función de decaimiento, donde la influencia decrece con el incremento de la distancia. Lo anterior permite pronosticar un determinado cambio de uso del suelo.

Los valores de las variables y los objetivos de cambio de uso del suelo son comúnmente cuantificados a partir de datos históricos obtenidos de imágenes satelitales o fotografías aéreas. Por otro lado, los algoritmos de transición espacial asumen explícitamente que las áreas vecinas influyen en la probabilidad de transición o cambio del área de cada celda o pixel de un mapa de uso de tipo raster. Los AC incorporan reglas simples acerca de los efectos de adyacencia espacial que gobiernan la dinámica del sistema y que dan lugar a patrones de comportamientos emergentes que usualmente son más complejos que aquellos generados por simples modelos de equilibrio (Theobald \& Hobbs, 1998).

En materia de hidrología, es necesario establecer correlaciones claras entre el cambio temporal del uso del suelo y el impacto que los parámetros de modelos hidrológicos puedan tener sobre los coeficientes de escorrentía. Las inundaciones en el 
mundo entero impactan más los centros de población que cualquier otro desastre natural (World Disasters Report, 2003). En este sentido, se ha demostrado que la forma más efectiva de reducir el riesgo de pérdidas humanas en zonas propensas a inundaciones es precisamente el desarrollo de sistemas de vigilancia y monitoreo, los cuales deben estar amparados en modelos hidrológicos/hidráulicos adecuadamente calibrados y validados (Negri et al., 2004).

Este trabajo pretende desarrollar una predicción del impacto del cambio temporal del uso del suelo sobre dos cuencas hidrológicas experimentales de alta pendiente. Para ello se utilizan los modelos MSSCUS del SIG IDRISI y el modelo hidrológico SWMM.

\section{Metodología}

Área de estudio

El área de estudio se ubica al noroeste de la provincia de Cartago, Costa Rica, e incluye las cuencas del río Toyogres y la quebrada Zopilote, con $14,6 \mathrm{~km}^{2}$ (Figura I).

La condición del terreno es abrupta, con un cambio de elevación en el orden de los 980 metros sobre el nivel del mar (msnm), desde las cercanías del volcán Irazú (2260 msnm) hasta el centro urbano de Cartago (I 280 msnm). La delimitación de ambas cuencas, así como la determinación de sus respectivas redes de drenaje y áreas tributarias, fueron previamente determinadas mediante funciones avanzadas de hidroprocesamiento, basadas en modelos de elevación de alta resolución (Méndez, 20 I2). No obstante, para efectos prácticos, se utilizó una cobertura de área mayor, de $4 \mathrm{~km}$ por 9,9 km $\left(39,6 \mathrm{~km}^{2}\right)$ con el fin de dar mayor libertad de interacción espacial a los algoritmos de clasificación del modelo MSSCUS.

\section{Descripción del Modelo SWMM}

El modelo hidrológico seleccionado para este caso de estudio es el modelo de gestión de aguas pluviales SWMM (Storm Water Management Model), el cual es un modelo de base física, totalmente distribuido para el análisis hidrológico e hidráulico de redes de drenaje esencialmente urbanas (Huber
\& Dickinson, 1988). Estructuralmente, el SWMM simula la respuesta de la cuenca a partir de datos de precipitación y parámetros físicos y numéricos relevantes.

Uno de los parámetros relevantes del modelo SWMM que está directamente relacionado con el coeficiente de escorrentía es el porcentaje de área impermeable (pimp). Dicho parámetro es determinante en la generación de escorrentía y representa el principal foco de atención de esta investigación. Para mayores detalles relacionados con la calibración y validación del modelo SWMM, ver Méndez (20|2).

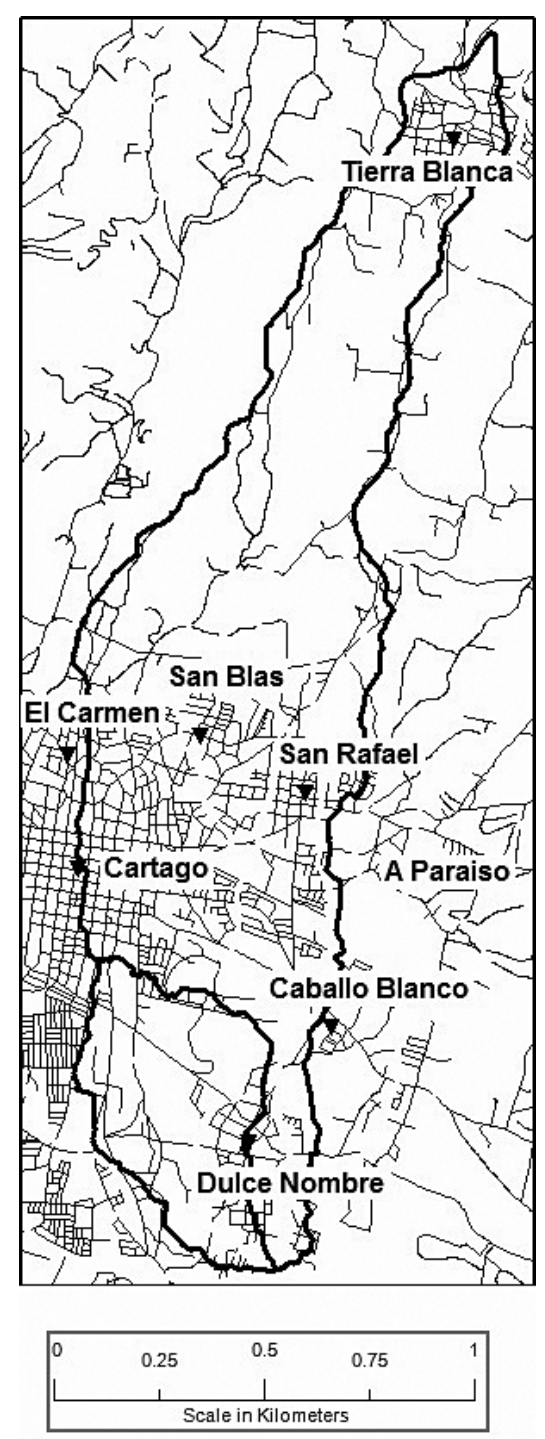

Figura I. Delimitación del área de trabajo para las cuencas del río Toyogres y la quebrada Zopilote. 
Descripción del modelo de cambio temporal de cobertura

El modelo de simulación simultánea de cambio de uso/cobertura del suelo (MSSCUS), basado en los planteamientos de las cadenas de Markov, Autómata Celular (AC) y evaluación multicriterio/ multiobjetivo (EMC) integrado en el SIG IDRISI fue utilizado para este propósito. El período de tiempo contemplado para esta modelación abarca desde 1997 hasta 201 I. Los registros históricos de 1997 corresponden a la misión TERRA financiada por RECOPE-MINAE (MINAE-CENIGA, 1998). A partir de allí, se ortorectificaron con base en un modelo de elevación digital (DEM) a 5m generado con los datos de la cartografía PRUGAM (PRUGAM, 2005) y procesado en el SIG ILWIS. El uso del suelo para 1997 se determinó readecuando las capas vectoriales (poligonales) de uso de suelo propuestas por el Plan Regional Urbano del Gran Área Metropolitana (PRUGAM) de Costa Rica en 2005. En ese sentido,

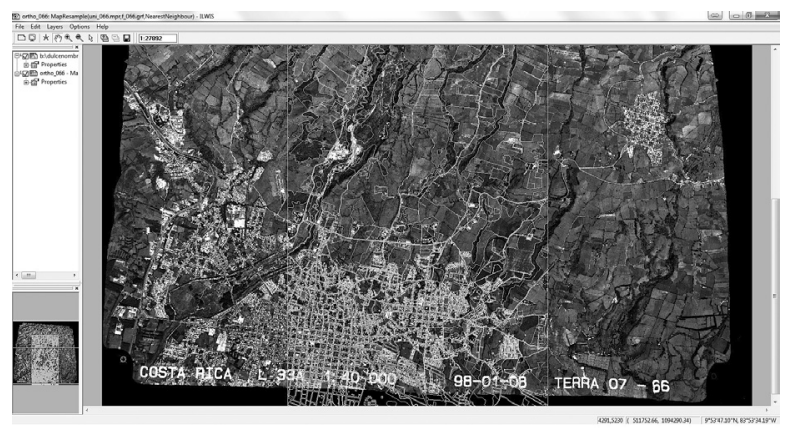

Figura 2. Comparación entre ortofotografía TERRA de 1997 y polígonos de uso (en blanco) de PRUGAM 2005. cada polígono de 2005 se comparó manualmente con su respectiva ortofotografía de 1997 y sus vértices readecuados para representar el uso que existía en el momento (Figura 2).

En el caso de los registros para 201 I, se utilizaron las imágenes ortorectificadas que se generaron a partir de las imágenes multiespectrales del satélite WorldView-2. El procedimiento de readecuación y consiguiente determinación del uso del suelo obedeció el mismo procedimiento utilizado para las imágenes de TERRA de 1997. Sin embargo, en el caso del satélite WorldView-2 se utilizaron principalmente las bandas infrarrojas para definir la clasificación de uso (Figura 3). Para efectos hidrológicos, la clasificación de uso contempló únicamente ocho clases relevantes para el modelo hidrológico SWMM con relación al parámetro pimp. A partir de la literatura se derivaron valores típicos de dicho parámetro en virtud de los usos del suelo considerados (Cuadro I).

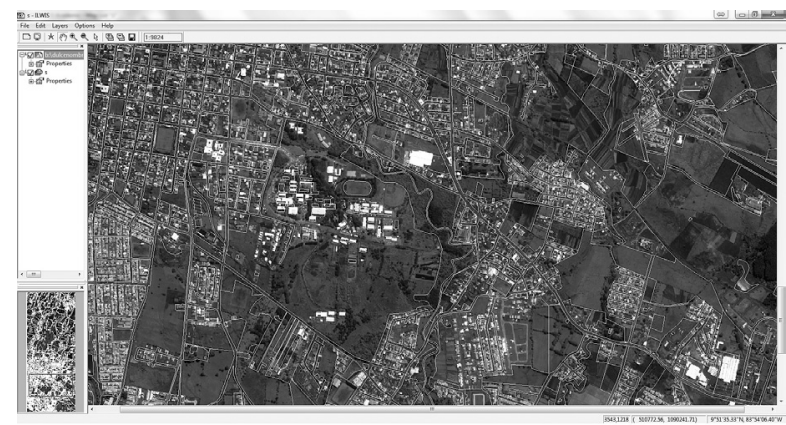

Figura 3. Comparación entre imágenes infrarrojas WorldView-2 de 201 I y polígonos de uso (en blanco) de PRUGAM 2005.

Cuadro I. Clasificación de uso utilizada en la parameterización del modelo SWMM.

\begin{tabular}{|c|c|c|c|}
\hline Tipo Clase & Nomeclatura & ID Clase & pimp (\%) * \\
\hline Residencial alta densidad & HDRES & I & 0,8 \\
\hline Residencial media densidad & MDRES & 2 & 0,6 \\
\hline Residencial baja densidad & LDRES & 8 & 0,3 \\
\hline Comercial & COMER & 3 & 0,85 \\
\hline Industrial & INDUS & 4 & 0,85 \\
\hline Tranporte & TRANS & 5 & 0,9 \\
\hline Protección & PROTEC & 6 & 0,05 \\
\hline Cultivos & CROPS & 7 & 0,05 \\
\hline
\end{tabular}

Fuente* Rossman 2007. 
Por otro lado, el modelo MSSCUS se ejecutó en tres fases generales. En la primera fase se determinó la probabilidad de transición de los diferentes usos/ objetivos para simular la situación al año 2025 sobre la base del periodo 1997-20 I I, mediante el método de las cadenas de Markov. Dado que cada uso en el periodo 1997-201 I, representa un objetivo en sí para el modelo Markov-CA-EMC, es necesario alimentarlo con factores y pesos relativos ligados a cada objetivo desde el punto de vista tipológico. El resultado de las cadenas de Markov son las matrices de probabilidad de transición, considerando los 14 años comprendidos entre 1997 y 201 I. Dichas matrices expresan la probabilidad de que un pixel de una categoría determinada cambie a otra clase o se mantenga constante en el próximo período de tiempo.

En la segunda fase se aplicó el método de evaluación multicriterio/multiobjetivo (EMC) incluido en IDRISI. Este método permite asignar los posibles usos/objetivos considerando un conjunto de factores de peso ponderados de acuerdo a su importancia relativa. El Cuadro 2 muestra los factores y restricciones que se utilizaron para cada objetivo. Es importante mencionar que los objetivos 9 (pastos) y 8 (invernaderos) finalmente fueron fundidos en el objetivo 7 (cultivos). Los factores de peso se centraron en los Índices de Fragilidad Ambiental (IFAS) adoptados por SETENA y PRUGAM y propuestos originalmente por el Dr. Allan Astorga (MINAE, 2008).

La propuesta de plan regulador para los cantones de Cartago, Oreamuno, Paraíso y el Guarco también se tomaron como factores de peso. Estos últimos, con mayor peso relativo que los IFAS dado que en sí incorporan el factor político-administrativo. Los factores corresponden a criterios que determinan la mayor o menor aptitud que tiene cada pixel para un uso de suelo/objetivo definido, se expresa a nivel de mapa raster en resoluciones de 8 bits ( 0 nula aptitud a 255 máxima aptitud). Algunos mapas son de carácter booleano (0 nula aptitud y I máxima aptitud). EI SIG IDRISI define estos mapas como mapas de "viabilidad" (suitability-maps en inglés).

La Figura 4 muestra los mapas booleanos y continuos para objetivos específicos (Cuadro 2). El método también considera restricciones geográficas (blanqueos) de carácter booleano, que limitan el análisis a un área geográfica particular.

En la última fase se ejecutó el modelo Autómata Celular (AC), tomando como datos de entrada las matrices de probabilidad de Markov y las EMC de cada uso/objetivo. El AC es un agente u objeto que tiene la habilidad de cambiar su estado, basado en la aplicación de una regla que relaciona el nuevo estado con su estado previo y la situación de pixeles vecinos (Eastman, 2003). Es un sistema dinámico discreto que se desarrolla en el espacio y el tiempo, compuesto por pixeles, cuadrículas, vecinos y reglas. Los vecinos son los pixeles que determinan el estado de una celda y las reglas definen el estado de la celda para el próximo período. En el caso de las simulaciones realizadas, se utilizó el vecino (o filtro) de Von Neumann, aplicado por defecto en el módulo CA_MARKOV de IDRISI.

\section{Resultados y análisis}

Cambio temporal de uso del suelo para el periodo I997- 20 I I

Durante el periodo 1997-20 I I (I4 años), el área de estudio experimentó cambios importantes sobre algunos de sus usos (Cuadro 3). No obstante, HDRES fue el uso que experimentó un mayor incremento, pasando de 6,18\% a 11,99\% del área total analizada $\left(39,6 \mathrm{~km}^{2}\right)$. Las figuras $5 \mathrm{~A}$ y $5 \mathrm{~B}$ evidencian una significativa densificación del casco central de Cartago (sector histórico) con una marcada expansión hacia el sector norte (San Blas), sector sur (Dulce Nombre y Agua Caliente) y sector sureste (Caballo Blanco), en algunos casos a manera de bloques aislados y discontinuos (Figura I).

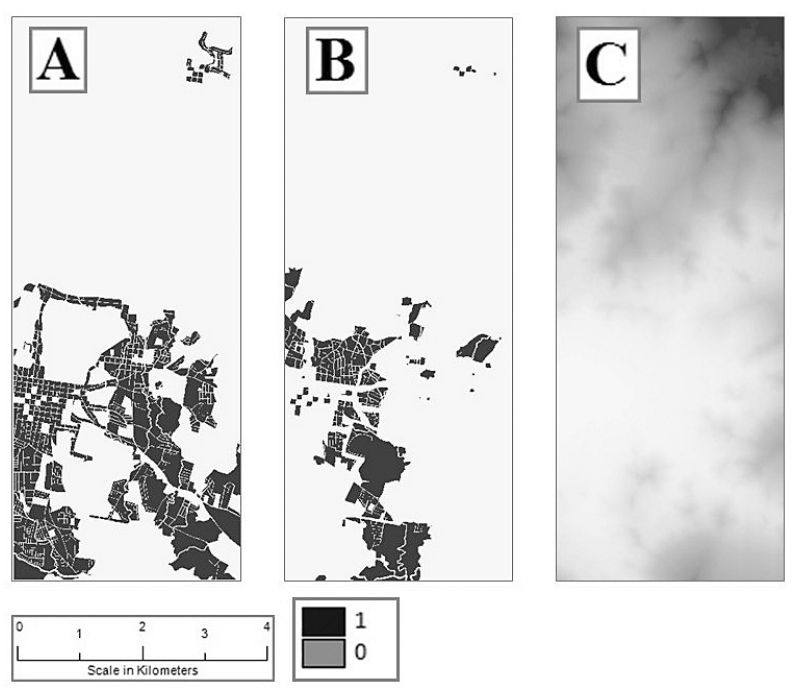

Figura 4. Mapas booleanos y continuos de factores asociados al modelo Markov-CA, HDRES (A), MDRES(B) y ROADDIST (C). 
Cuadro 2. Factores de peso y objetivos utilizados en el modelo Markov-AC.

\begin{tabular}{|c|c|c|c|c|}
\hline Tipo Clase & Nomeclatura & Resolución (bits) & Factores & Restricciones \\
\hline \multirow{7}{*}{ Residencial alta densidad } & \multirow{7}{*}{ HDRES } & 0_255 & IFA_GEO_RES & WATERCON \\
\hline & & 0_255 & IFA_BIO & HDLANDCON \\
\hline & & 0_255 & IFA_EDA & PROTECCON \\
\hline & & 0_255 & IFA_ANTRO & \\
\hline & & metros & ROADDIST & \\
\hline & & minutos & TOWNDIST & \\
\hline & & $0 \_255$ & HDRECLASS & \\
\hline \multirow{7}{*}{ Residencial media densidad } & \multirow{7}{*}{ MDRES } & 0_255 & IFA_GEO_RES & WATERCON \\
\hline & & $0 \_255$ & IFA_BIO & MDLANDCON \\
\hline & & 0_255 & IFA_EDA & PROTECCON \\
\hline & & $0 \_255$ & IFA_ANTRO & \\
\hline & & metros & ROADDIST & \\
\hline & & minutos & TOWNDIST & \\
\hline & & $0 \_255$ & MDRECLASS & \\
\hline \multirow{3}{*}{ Comercial } & \multirow{3}{*}{ COMER } & 0_255 & MASTER_COMER & WATERCON \\
\hline & & & & \\
\hline & & & & PROTECCON \\
\hline \multirow{3}{*}{ Industrial } & \multirow{3}{*}{ INDUS } & 0_255 & MASTER_INDUS & WATERCON \\
\hline & & & & GELANDCON \\
\hline & & & & PROTECCON \\
\hline Transporte & TRANS & 0_255 & WorldView-2 & \\
\hline \multirow{4}{*}{ Protección } & \multirow{4}{*}{ PROTEC } & 0_255 & IFA_GEO_PRO & \\
\hline & & 0_255 & IFA_BIO & PROTECCON \\
\hline & & 0_255 & IFA_EDA & \\
\hline & & 0_255 & IFA_ANTRO & \\
\hline \multirow{5}{*}{ Cultivos } & \multirow{5}{*}{ CROPS } & 0_255 & IFA_GEO_AGRI & WATERCON \\
\hline & & $0 \_255$ & IFA_BIO & GELANDCON \\
\hline & & 0_255 & IFA_EDA & PROTECCON \\
\hline & & 0_255 & IFA_ANTRO & \\
\hline & & minutos & TOWNDIST & \\
\hline Invernaderos & GREEN & BOOLEAN & AGUA & \\
\hline \multirow{7}{*}{ Residencial baja densidad } & \multirow{7}{*}{ LDRES } & 0_255 & IFA_GEO_RES & WATERCON \\
\hline & & 0_255 & IFA_BIO & LDLANDCON \\
\hline & & 0_255 & IFA_EDA & PROTECCON \\
\hline & & 0_255 & IFA_ANTRO & \\
\hline & & metros & ROADDIST & \\
\hline & & minutos & TOWNDIST & \\
\hline & & 0_255 & LDRECLASS & \\
\hline \multirow{5}{*}{ Pastos } & \multirow{5}{*}{ PASTURE } & 0_255 & IFA_GEO_AGRI & WATERCON \\
\hline & & $0 \_255$ & IFA_BIO & GELANDCON \\
\hline & & 0_255 & IFA_EDA & PROTECCON \\
\hline & & 0_255 & IFA_ANTRO & \\
\hline & & minutos & PLANTDIST & \\
\hline
\end{tabular}


Continuación

\begin{tabular}{|l|l|}
\hline \multicolumn{1}{|l|}{ Nomenclatura: } & \\
\hline IFA_GEO_RES: & IFA de geoaptitud \\
\hline IFA_BIO: & IFA de bioaptitud \\
\hline IFA_EDA: & IFA de edafoaptitud \\
\hline IFA_ANTRO: & IFA de antropoaptitud \\
\hline ROADDIST: & Distancia a red de transporte \\
\hline TOWNDIST: & Distancia a centro de población \\
\hline WATERCON: & Restricción de expansión en cuerpos de agua \\
\hline PROTECCON: & Restricción de expansión enzonas de protección \\
\hline
\end{tabular}

Cuadro 3. Evolución temporal de los usos del suelo para el sector central de Cartago para el periodo 1997- 201।.

\begin{tabular}{|c|c|c|c|c|c|}
\hline \multirow{2}{*}{ USO } & \multicolumn{2}{|c|}{1997} & \multicolumn{2}{|c|}{2011} & \multirow{2}{*}{$\begin{array}{c}\text { Cambio 1997-20 I I } \\
\text { D\% Relativo }\end{array}$} \\
\hline & Area $(\mathrm{Ha})$ & $\%$ Area total & Area $(\mathrm{Ha})$ & $\%$ Area total & \\
\hline HDRES & 245,09 & 6,18 & 475,21 & 11,99 & 5,81 \\
\hline MDRES & 212,44 & 5,36 & 310,12 & 7,83 & 2,47 \\
\hline COMER & 15,30 & 0,39 & 10,38 & 0,26 & $-0,12$ \\
\hline INDUS & 43,96 & 1,11 & 49,80 & 1,26 & 0,15 \\
\hline TRANS & 240,47 & 6,07 & 264,88 & 6,68 & 0,62 \\
\hline PROTEC & $47 \mid, 49$ & 11,90 & 460,87 & 11,63 & $-0,27$ \\
\hline CROPS & 2418,99 & 61,04 & 2148,24 & 54,21 & $-6,83$ \\
\hline LDRES & 315,04 & 7,95 & 243,27 & 6,14 & $-|, 8|$ \\
\hline TOTAL & 3962,78 & 100,00 & 3962,78 & 100,00 & \\
\hline
\end{tabular}

En otros casos (como Dulce nombre y Agua Caliente), los patrones resultan más sistemáticos y ordenados, principalmente debido a grandes proyectos de interés social. El crecimiento en el sector histórico podría deberse esencialmente a la renovación de estructuras preexistentes y la segregación de propiedades relativamente grandes en otras más pequeñas. Este fenómeno de aglomeración lleva a que se cubra gran parte (si no toda) del área/ terreno disponible. MDRES tuvo un D\% relativo del $2,47 \%$ que en buena medida concuerda con las disminuciones de - I,81\% y -0,27\% correspondientes a LDRES y PROTEC respectivamente.

En términos generales, el crecimiento de las áreas ligadas directamente a usos residenciales en los últimos 14 años ha sido importante para la ciudad de Cartago. Si bien es cierto el área actualmente contemplada no cubre en su totalidad los cuatro municipios originalmente incluidos (Cartago, Oreamuno, Paraíso y el Guarco), sí arroja una idea clara de la situación general de esta sección del Gran
Area Metropolitana (GAM). El aumento porcentual sobre estos usos y la disminución de aquellos con mayor facultad de compensación hidrológica (por ej., CROPS con D\% relativo del $-6,83 \%)$, tienen

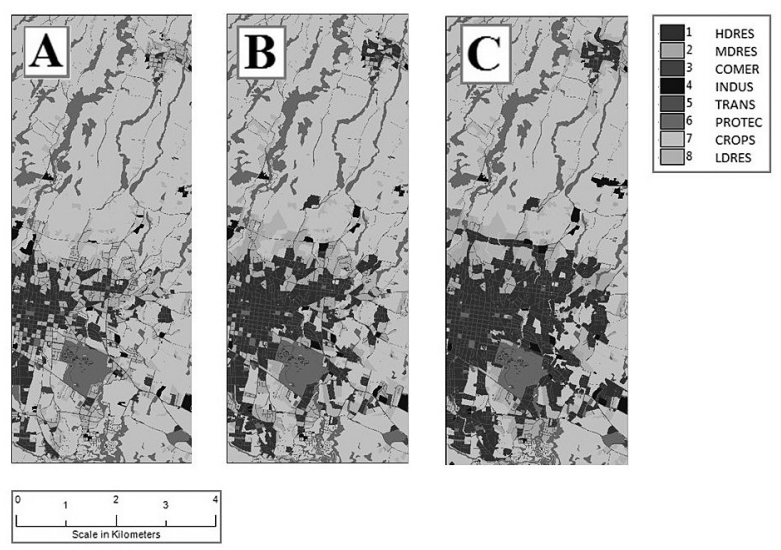

Figura 5. Cambio temporal de uso del suelo propuesto para 2025 (C) con base en los registros históricos de 1997 (A) y 201 I (B). 
importantes repercusiones sobre los coeficientes de escorrentía de las cuencas bajo estudio. En este sentido, se nota también una leve disminución sobre las áreas de protección (PROTEC), pasando de 1 I,90\% a 1 1,63\% del área total contemplada, lo que a la vez podría indicar invasión de las áreas de protección de ríos y quebradas.

\section{Cadenas de Markov}

A partir de registros temporales disponibles para el periodo 1997-20II, se procedió a ejecutar el módulo de cadenas de Markov de IDRISI-GIS, con lo que se obtuvieron las matrices de probabilidad de transición para el año 2025 del área bajo estudio (Cuadro 4). Estas matrices representan la base cuantitativa de las estimaciones futuras de cambio de uso/cobertura. En este sentido, resulta interesante notar que existe una probabilidad del $100 \%$ de que HDRES siga siendo HDRES, o lo que es lo mismo, no hay probabilidad de que cambie a ningún otro uso. Por su parte, MDRES tiene un 54,2\% de probabilidad de convertirse en HDRES y tan solo un 7\% de pasar a LDRES.

Por su parte, LDRES tiene un 17,2\% de probabilidad de pasar a HDRES y un $46,6 \%$ de pasar a MDRES. Estos tres usos son sin duda los de mayor impacto a nivel de generación de escorrentía. CROPS parece variar muy poco, con saltos pequeños de $2,4 \%$ y 2,3\% a HDRES y MDRES respectivamente. Por el contrario, parece haber una tendencia de cambio a LDRES, con cúmulos de casas dispersas principalmente en el sector norte de Tierra Blanca (Figura 5C). El resto de los usos experimenta cambios muy sutiles que podrían perfectamente estar dentro de la incertidumbre espacial de los datos utilizados.

Debe tomarse en cuenta que aunque la resolución espacial seleccionada fue de $2 \mathrm{~m}$, la resolución de las imágenes TERRA de 1997 fue de 3,6 m, por lo que

Cuadro 4. Probabilidades de transición con base en las cadenas de Markov para los usos del suelo en el sector central de Cartago para el periodo 201 I-2025.

\begin{tabular}{|c|c|c|c|c|c|c|c|c|c|c|}
\hline \multirow{2}{*}{\multicolumn{2}{|c|}{$\begin{array}{l}\text { USO } \\
\text { HDRES }\end{array}$}} & \multicolumn{9}{|c|}{ Periodo actual a 2011} \\
\hline & & MDRES & COMER & INDUS & TRANS & PROTEC & CROPS & LDRES & TOTAL & \\
\hline \multirow{8}{*}{ 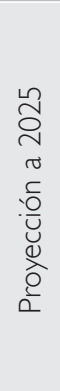 } & HDRES & 1,000 & 0,000 & 0,000 & 0,000 & 0,000 & 0,000 & 0,000 & 0,000 & 1,000 \\
\hline & MDRES & 0,542 & 0,451 & 0,000 & 0,000 & 0,000 & 0,000 & 0,000 & 0,007 & 1,000 \\
\hline & COMER & 0,000 & 0,272 & 0,679 & 0,000 & 0,000 & 0,000 & 0,049 & 0,000 & 1,000 \\
\hline & INDUS & 0,009 & 0,144 & 0,000 & 0,832 & 0,000 & 0,000 & 0,000 & 0,014 & 1,000 \\
\hline & TRANS & 0,000 & 0,000 & 0,000 & 0,000 & 1,000 & 0,000 & 0,000 & 0,000 & 1,000 \\
\hline & PROTEC & 0,005 & 0,001 & 0,000 & 0,000 & 0,012 & 0,968 & 0,000 & 0,014 & 1,000 \\
\hline & CROPS & 0,024 & 0,023 & 0,000 & 0,005 & 0,008 & 0,001 & 0,888 & 0,051 & 1,000 \\
\hline & LDRES & 0,172 & 0,466 & 0,000 & 0,006 & 0,000 & 0,003 & 0,000 & 0,353 & 1,000 \\
\hline
\end{tabular}

Cuadro 5. Número de pixeles estimados a cambiar entre los usos del suelo en el sector central de Cartago para el periodo 201 I-2025.

\begin{tabular}{|c|c|c|c|c|c|c|c|c|c|}
\hline \multirow{2}{*}{\multicolumn{2}{|c|}{$\begin{array}{c}\text { USO } \\
\text { HDRES }\end{array}$}} & \multicolumn{8}{|c|}{ Periodo actual a 2011} \\
\hline & & MDRES & COMER & INDUS & TRANS & PROTEC & CROPS & LDRES & \\
\hline \multirow{8}{*}{ 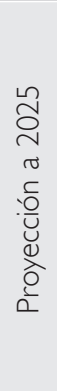 } & HDRES & 1188030 & 0 & 0 & 0 & 0 & 0 & 0 & 0 \\
\hline & MDRES & 420437 & 349484 & 0 & 53 & 0 & 0 & 47 & 5292 \\
\hline & COMER & 0 & 7061 & 17609 & 0 & 0 & 0 & 1279 & 0 \\
\hline & INDUS & 1167 & 17930 & 0 & 103636 & 0 & 0 & 0 & 1767 \\
\hline & TRANS & 0 & 0 & 0 & 2 & 662189 & 0 & 0 & 0 \\
\hline & PROTEC & 5128 & 1376 & 0 & 0 & 13655 & | | | 56 | & 0 & 16265 \\
\hline & CROPS & 129647 & 125773 & 0 & 24799 & 41775 & 7205 & 4767775 & 273623 \\
\hline & LDRES & 104284 & 283123 & 0 & 3903 & 0 & 2011 & 0 & 214866 \\
\hline
\end{tabular}


existe una evidente generalización de la información y pérdida de detalle. Lo anterior también se aplica a la mayoría de los mapas de viabilidad mostrados por el Cuadro 2, que incluye los IFAS y las capas espaciales de los planes reguladores propuestas por PRUGAM para los municipios involucrados. Algunas de estas capas presentan resoluciones espaciales de 1:10 000, |:50 000 e incluso 1:200 000, lo cual evidentemente no compite con la resolución espacial de las imágenes WorldView-2. El Cuadro 5 transforma la matriz de probabilidades a una matriz cuantitativa de cambio de cobertura a nivel de píxel, lo que finalmente se traduce en área efectiva por uso.

\section{Aplicación de Autómatas Celulares}

Los registros históricos, junto con la proyección a 2025 de cambio de cobertura para el área de estudio, se muestran en la Figura 5. Los resultados de la simulación reafirman las tendencias observadas en el periodo 1997-201 I, donde la proyección mayor de crecimiento se centra esencialmente en HDRES y LDRES, con consistentes disminuciones de MDRES y CROPS (Cuadro 6). Aparte de la densificación del centro histórico de Cartago, puede verse un marcado crecimiento de HDRES a lo largo del eje vial que conduce a Tierra Blanca (Figura 5C). Lo mismo pareciera ocurrir a lo largo del eje vial que comunica Cartago con Paraíso (Figura 5C). La Figura 6 muestra un resumen del comportamiento de los diferentes usos desde 1997 hasta su proyección en 2025.

Cuadro 6. Proyección de la evolución temporal de los usos del suelo para el sector central de Cartago para el periodo 20II-2025.

\begin{tabular}{|c|c|c|c|c|c|}
\hline \multirow{2}{*}{ USO } & \multicolumn{2}{|c|}{$201 \mathrm{I}$} & \multicolumn{2}{c|}{2025} & Cambio 20 I I-2025 \\
\cline { 2 - 6 } & Area $(\mathrm{Ha})$ & $\%$ & Area $(\mathrm{Ha})$ & $\%$ & D\% Relativo \\
\hline HDRES & 475,21 & 11,99 & 727,76 & 18,36 & 6,37 \\
\hline MDRES & 310,12 & 7,83 & 252,09 & 6,36 & $-1,46$ \\
\hline COMER & 10,38 & 0,26 & 9,10 & 0,23 & $-0,03$ \\
\hline INDUS & 49,80 & 1,26 & 52,96 & 1,34 & 0,08 \\
\hline TRANS & 264,88 & 6,68 & 274,80 & 6,93 & 0,25 \\
\hline PROTEC & 460,87 & 11,63 & 450,57 & 11,37 & $-0,26$ \\
\hline CROPS & 2148,24 & 54,21 & 1913,98 & 48,30 & $-5,91$ \\
\hline LDRES & 243,27 & 6,14 & 287,24 & 7,25 & 1,11 \\
\hline TOTAL & 3962,78 & 100,00 & 3968,50 & 100,00 & \\
\hline
\end{tabular}

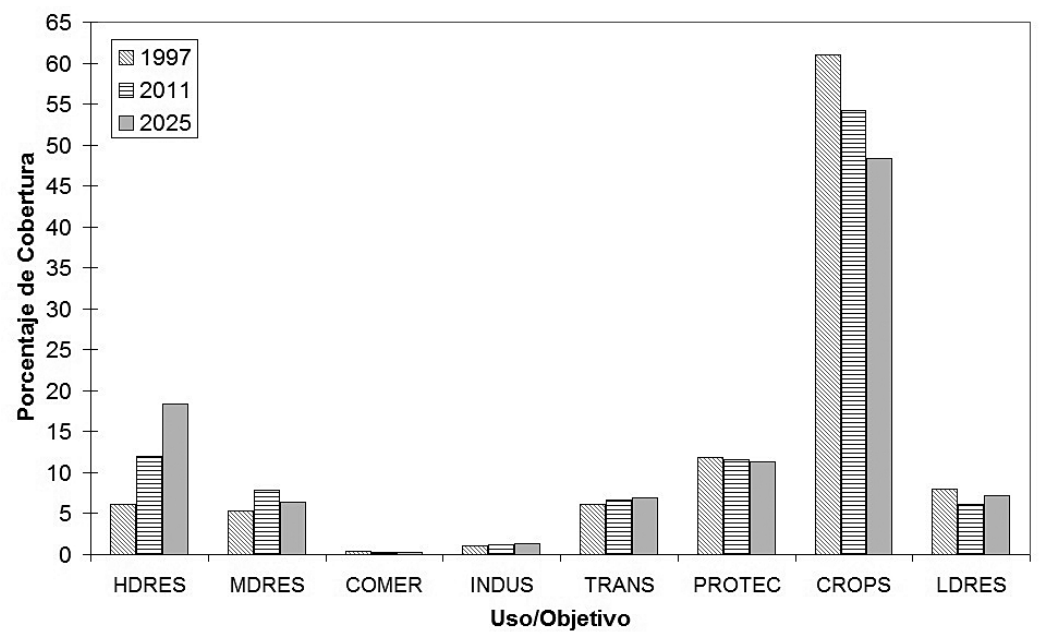

Figura 6. Evolución temporal de los usos del suelo para el sector central de Cartago para los años 1997, 201 I y 2025. 
De nuevo se puede apreciar que la tendencia de cambio se centra sobre los usos residenciales en sacrificio del uso agrícola primordialmente, mientras que el resto de los usos experimenta cambios leves.

Evaluación y simulación del cambio en el coeficiente de escorrentía superficial

Dado que la validación y calibración del modelo SWMM se realizó con tormentas reales y observaciones reales de flujo, se pretende evaluar el impacto que el cambio del coeficiente de escorrentía superficial pudiese tener sobre la respuesta real de

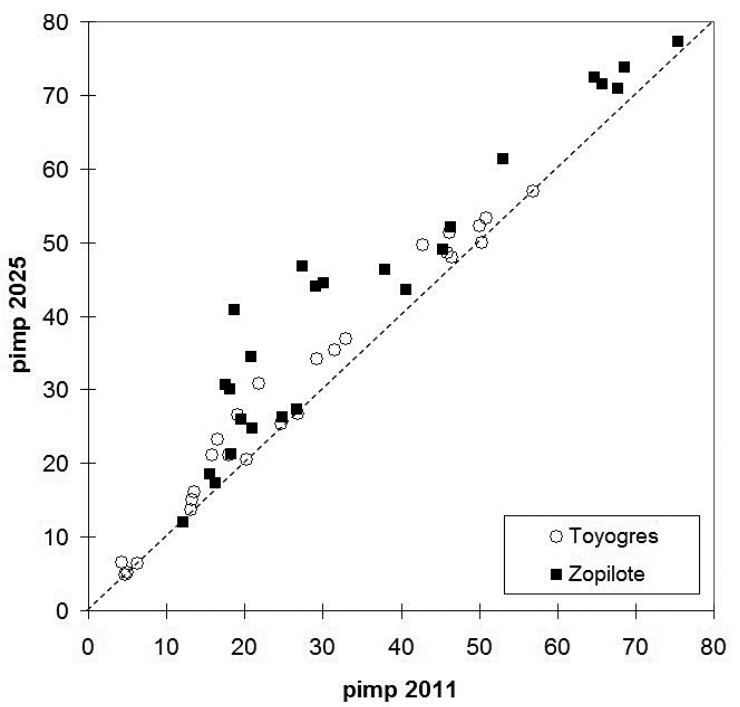

Figura 7. Correlación temporal propuesta del parámetro pimp para el periodo 20 I -2025. las cuencas contempladas en este estudio (Méndez, 20I2). En virtud de lo señalado y en relación con la estructura interna del modelo SWMM, el parámetro clave a evaluar es precisamente el porcentaje de área impermeable o pimp, ya que con base en el análisis de sensibilidad, este no guarda mayor correlación con el resto de los parámetros (Méndez, 2012).

La Figura 7 muestra la correlación temporal propuesta del parámetro pimp para el periodo 20 I I-2025. Es evidente que la tendencia al crecimiento es mucho más marcada para la cuenca del Zopilote que para la cuenca del Toyogres.

Con el propósito de realizar una simulación temporal de las consecuencias que este aumento del parámetro pimp podría tener en ambas cuencas, se decidió utilizar una de las tormentas reales usadas en la calibración y validación del modelo SWMM (Méndez, 20I2). Dicha tormenta, conocida como T5 y ocurrida el 2 de octubre de 201 I, alcanzó un volumen máximo de $53,56 \mathrm{~mm}$ y una intensidad máxima de $120 \mathrm{~mm} / \mathrm{hr} / 5 \mathrm{~min}$. La razón para utilizar una tormenta real radica en la dificultad de apegarse a tormentas sintéticas, cuyo comportamiento no refleja en lo absoluto las particularidades de una tormenta a escala real.

En este sentido, SWMM fue nuevamente cargado con el promedio simple de los parámetros calibrados y se actualizó el valor de pimp para cada subcuenca contemplada en 2025. El hidrograma presentado en la Figura 8 muestra el cambio proporcional que

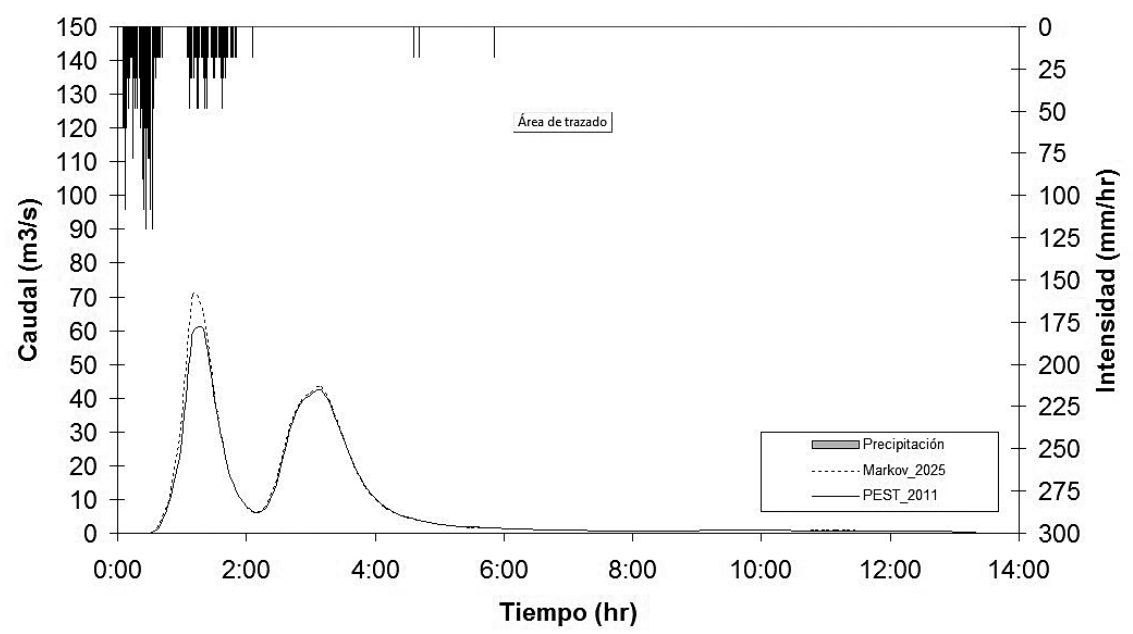

Figura 8. Comparación entre los hidrogramas modelados por SWMM para la tormenta T5 para los años 20 II y 2025 en la cuenca del río Toyogres. 
exhibiría la cuenca del río Toyogres ante el eventual aumento de pimp para 2025.

Queda claro que la afectación directa es sobre el caudal pico, pasando de $60,4 \mathrm{~m}^{3} / \mathrm{s}$ en 201 I a 70,6 $\mathrm{m}^{3} / \mathrm{s}$ en 2025 , o sea, un aumento relativo de $14,5 \%$. Desde el punto de vista hidráulico, este aumento no compromete la red de drenaje del río Toyogres en ninguna de las secciones caracterizadas en campo, por lo menos para una tormenta igual o menor a T5.

La situación es similar para la cuenca de la quebrada Zopilote (Figura 9), donde el caudal pico pasaría de $10,6 \mathrm{~m}^{3} / \mathrm{s}$ a $14,3 \mathrm{~m}^{3} / \mathrm{s}$, o lo que es lo mismo, un aumento relativo de $25,9 \%$ a 2025 . Queda claro también que el volumen escurrido aumentaría considerablemente, casi de forma proporcional en virtud de lo mostrado. En resumen, si bien es cierto el aumento en el porcentaje de área impermeable, consecuencia del aumento proporcional sobre pimp, podría parecer relativamente alto en la cuenca del Toyogres, el aumento sobre su caudal pico no es tan importante, en el tanto en que los demás usos de carácter meramente permeable (crops y protect, principalmente) con gran capacidad de almacenamiento permanecen relativamente constantes. Consecuentemente, tiende a haber un proceso de compensación o buffer de la escorrentía. La preocupación mayor se centra sobre la red de drenaje de la quebrada Zopilote, la cual sí experimenta un incremento considerable en el caudal pico y el volumen escurrido.
Bajo cualquier circunstancia, debe quedar claro que la proyección de cambio de uso propuesta por el modelo MSSCUS de IDRISI es tan solo una simulación y depende de que los factores de peso incluidos, especialmente las propuestas del plan regulador de los municipios involucrados, se pongan en práctica.

\section{Conclusiones}

El modelo de simulación simultánea de cambio de uso/cobertura de suelo (MSSCUS) de IDRISIANDES, basado en las cadenas de Markov, Autómata Celular (AC) y evaluación multicriterio/multiobjetivo (EMC), fue utilizado para predecir el impacto del cambio temporal del uso del suelo al año 2025 sobre dos cuencas hidrológicas experimentales de alta pendiente en Costa Rica.

Las cuencas pertenecen al río Toyogres y la quebrada zopilote, ambas ubicadas en el perímetro urbano de la ciudad de Cartago. El modelo hidrológico SWMM fue calibrado y validado con tormentas reales captadas durante $201 \mathrm{I}$ con el fin de servir de plataforma de análisis hidrológico. Los registros históricos utilizados tanto en MSSCUS como en SWMM se fundamentaron en fotografías áreas de la misión TERRA de 1997 e imágenes satelitales multiespectrales del satélite WorldView-2 tomadas en 20I I. Con base en las proyecciones temporales arrojadas por los modelos MSSCUS y SWMM, se pudo determinar que para el año 2025, el aumento sobre el caudal pico para una tormenta de alta

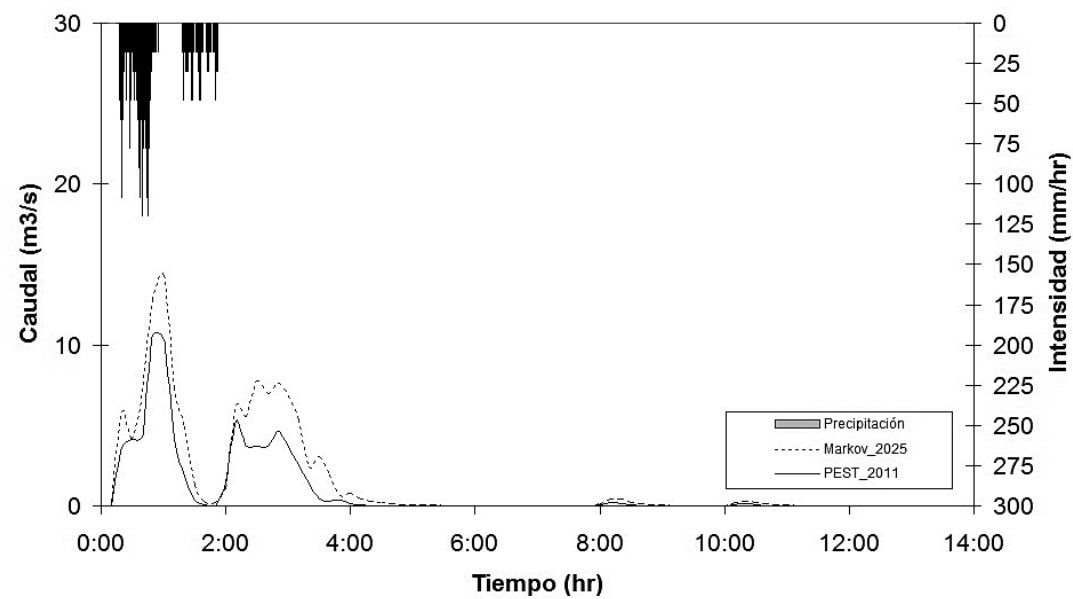

Figura 9. Comparación entre los hidrogramas modelados por SWMM para la tormenta T5 para los años 201 I y 2025 en la cuenca de la quebrada Zopilote. 
intensidad como T5 sería de alrededor del 15\% para el cauce del río Toyogres y de $26 \%$ para el cauce de la quebrada Zopilote, siendo este último de gran relevancia.

Lo anterior se da como consecuencia de un aumento del área impermeable en relación con el aumento del porcentaje de área impermeable (pimp), parámetro clave en los procesos de generación de escorrentía. Los usos/objetivos que sufren mayores cambios en la cuenca de la quebrada zopilote son HDRES, MDRES, LDRES, y CROPS. Queda claro que la red de drenaje de esta cuenca exhibe serias limitaciones, que aun mediante la aplicación de tormentas sintéticas presenta una agravada incapacidad de tránsito hidráulico.

Finalmente, la combinación de modelos MSSCUS y modelos hidrológicos e hidráulicos abre nuevas posibilidades en la exploración del impacto ambiental que el crecimiento urbano de las ciudades ejerce sobre los sistemas naturales. Deben igualmente reconocerse las limitaciones que las proyecciones de nuestros modelos puedan tener ante la ausencia de información necesaria en Icalibración y validación de estos.

\section{Bibliografía}

Brockerhoff, M.P. (2000). An urbanizing world. Pop. Bull. 55, 3-44.

Dongquan, Z., Jining, C., Haozheng, W., Qingyuan, T., Shangbing, C. \& Zheng, S. (2009). GIS-based urban rainfall-runoff modeling using an automatic catchment-discretization approach: a case study in Macau. Environmental Earth Sciences, Vol. 59, No. 2, 465-472.

Du, J.K., Xie, S.P., Xu, Y.P., Xu, C.Y. \& Singh,V.P. (2007). Development and testing of a simple physically-based distributed rainfallrunoff model for storm runoff simulation in humid forested basins. J. Hydrol. 306: 334-346.

Eastman, J. (20I0). IDRISI Andes. Guide to GIS and Image Processing. Worcester,

MA: Clark Labs, Clark University.

Forte, F., Strobl, R.O. \& Pennetta, L. (2006). A methodology using GIS, aerial photos and remote sensing for loss estimation and flood vulnerability analysis in the Supersano-RuffanoNociglia Graben, southern Italy. Environmental Geology (50)4: 58|-594.
Goudie, A. (1990). The human impact on the natural environment. 3a. ed. Cambridge, Massachusetts: The MIT Press.

Henríquez, C., Azócar, G. \& Aguayo, M. (2006). Cambio de uso del suelo y escorrentía superficial: aplicación de un modelo de simulación espacial en Los Ángeles, VIII Región del Biobío, Chile. Revista de Geografía Norte Grande Nº 36, p. 6I-74.

Huber, W. \& Dickinson, R. (1988). Storm Water Management Model Version 4, Part A: User's Manual. EPAl60013-88100 Ia. US Environmental Protection Agency.

International Federation of Red Cross and Red Crescent Societies (2003). World Disasters Report. p. 239.

Méndez, M. (20/2). Calibración y validación del modelo hidrológico SWMM en cuencas hidrológicas de alta pendiente. Revista Tecnología en Marcha. Artículo en prensa.

MINAE (2008). Manual de Instrumentos Técnicos para el proceso de Evaluación de Impacto Ambiental (Manual de EIA). Decreto No 32967-MINAE.

MINAE-CENIGA (1998). Información Cartografica de la Mision TERRA-Costa Rica. Ministerio de Ambiente, Energía y Minas.

Negri, A., Burkardt, N., Golden, J.H., Halverson, J.B., Huffman, G.J., Larsen, M.C., Mcginley, J.A., Updike, R.G., Verdin, J.P. \& Wieczorek, J.F. (2004). The Hurricane-Flood-Landslide Continuum. Bulletin of American Meteorological Society (doi: | 0.1 |75/BAMS-86-9-|24I).

Rossman, L.A. (2007). Stormwater Management Model User's Manual, Version 5.0. U.S. Environmental Research Agency. EPA/600/R-05/040 Revised June 2007.

Seth, I., Soonthornnonda, P. \& Christensen, E.R. (2006). Use of GIS in urban storm-water modeling. J. Environ. Eng. 32(12): |550-1552.

Theobald, D. \& Hobbs, N. (1998). Forecasting rural land-use change: a comparison of regression and spatial transitionbased models. Geographical and Environmental Modelling $\mathrm{N}^{\circ}$ 2, Vol. I, p. 62-82.

Weng, Q. (200I). Modeling Urban Growth Effects on Surface Runoff with the Integration of Remote Sensing and GIS. Environmental Management 28(6): 737-748.

Yu, P.S., Yang, T. \& Chen, S.J. (200 I). Comparison of uncertainty analysis methods for a distributed rainfall-runoff model. J. Hydrol. 244: 43-59.

Yuan, F., Sawaya, K., Loeffelholz, B. \& Bauer, M. (2005). Land cover classification and change analysis of the Twin cities (Minnesota) Metropolitan Area by multitemporal Landsat Remote Sens. Environ. Remote Sens. Environ 98, 317-328.

Zhou, G. \& Liebhold, A. ( 1995). Forecasting the Spatial Dynamics of Gypsy-Moth Out-breaks Using CellularTransition Models. Landscape Ecology No 10, Vol. 3, p. 177-189. 